\title{
Macroscopic and Microscopic Study of a CePdIn Compound
}

\author{
M. Klicpera ${ }^{a, b, *}$, P. Javorskŕ ${ }^{a}$, I. Puente-Orench ${ }^{b, c}$ And A. Hoser ${ }^{d}$ \\ ${ }^{a}$ Charles University in Prague, Faculty of Mathematics and Physics, Department of Condensed Matter Physics, \\ Ke Karlovu 5, 12116 Prague 2, Czech Republic \\ ${ }^{b}$ Institut Laue-Langevin, 71 av. des Martyrs, CS 20156, 38042 Grenoble Cedex 9, France \\ ${ }^{c}$ Instituto de Ciencia de Materiales de Aragón, CSIC, Pedro Cerbuna 12, 50009 Zaragoza, Spain \\ ${ }^{d}$ Helmholtz-Zentrum Berlin für Materialien und Energie GmbH, Hahn-Meitner-Platz 1, 14-109 Berlin, Germany
}

\begin{abstract}
The magnetization and electrical resistivity measurements on a CePdIn single crystal as well as its preparation and structural characterization are presented. The negative paramagnetic Curie temperatures indicate antiferromagnetic ground state, the anisotropy of the paramagnetic Curie temperature amounts $22.7 \mathrm{~K}$. No ferromagnetic correlations were indicated. Powder neutron diffraction experiment performed at temperatures down to $0.4 \mathrm{~K}$ did not lead to observation of any magnetic peak in diffraction patterns. We estimate the magnetic moment on Ce atoms to be significantly lower than $0.5 \mu_{\mathrm{B}}$. The temperature development of lattice parameters documents the standard thermal expansion of the unit cell; no signs of structural phase transition were observed.
\end{abstract}

DOI: 10.12693/APhysPolA.131.970

PACS/topics: $61.05 . \mathrm{F}-$, 71.27.+a, 72.15.Eb

\section{Introduction}

The ternary hexagonal CeTIn compounds, where $\mathrm{T}=\mathrm{Ni}, \mathrm{Pd}, \mathrm{Pt}$, crystallizing in ZrNiAl-type structure (space group $P-62 m$ ) [1], attract the attention already many years. The electronic properties depend strongly on $d$-element $\mathrm{T}$ despite the fact that this substitution is isostructural and isoelectronic, i.e. only the character of $d$-electrons and lattice parameters are changed. CeNiIn is a valence fluctuating compound without magnetic order, at least down to $50 \mathrm{mK}$, and with moderately enhanced electronic specific heat at low temperatures [2]. The antiferromagnetic order with the Néel temperature $T_{\mathrm{N}}=1.7 \mathrm{~K}$ was observed in CePdIn $[3,4]$. CePtIn with paramagnetic ground state and anomalous specific heat development at low temperatures stands on the border between CeNiIn and CePdIn counterparts $[1,2,5,6]$.

Antiferromagnetically ordered CePdIn reveals additional anomaly on specific heat data at $T_{A}=0.9 \mathrm{~K}$ [3]. The presence of this anomaly has not been explained so far. The suspicion on further magnetic phase transition was reported, however no microscopic evidence has been obtained. The substitution of Pd by a few percent of $\mathrm{Rh}$ [7], Ni [8], or Pt [9] results in a usual one-peak anomaly in specific heat. With $\mathrm{Ni}$ (or Rh or Pt) substitution the Ruderman-Kittel-Kasuya-Yoshida (RKKY) interaction in the CePdIn compound is weakening and with a $40 \%$ of Ni content the Kondo interaction dominates and $\mathrm{nFL}$ or rather anomalous $\mathrm{nFL}$ behavior occurs [8]. The value of $C / T \approx 0.7 \mathrm{~J} \mathrm{~K}^{-2} \mathrm{~mol}^{-1}$ at temperature of $60 \mathrm{mK}$ observed in CePdIn [3] classifies the compound as a heavy-fermion system.

*corresponding author; e-mail: mi.klicpera@seznam.cz
Previous studies on CePdIn were focused on temperature development of physical properties. The present single crystal study brings additional information on magnetization and mainly electrical resistivity in applied magnetic field. The powder neutron diffraction data reveal the temperature evolution of lattice parameters in CePdIn.

\section{Experimental methods}

Single crystalline CePdIn was prepared by the Czochralski growth method using the tri-arc furnace under protection of an argon atmosphere. The quality and crystal symmetry were verified by both X-ray powder and single crystal Laue diffraction. The chemical composition was checked using scanning electron microscope equipped with an energy dispersive X-ray (EDX) analyzer.

The MPMS and PPMS (magnetic/physical property measurement system, Quantum Design) were used for the magnetization and electrical resistivity measurements, respectively. The same prism-shape samples (with longedge cut along and perpendicular to the $c$-axis) with mass $\approx 30 \mathrm{mg}$ were used for both types of measurement.

The powder neutron diffraction experiments at temperatures from 0.4 to $250 \mathrm{~K}$ were performed using focusing powder diffractometer E6 at Helmholz Zentrum Berlin, Germany $(\lambda=2.443 \AA)$ and thermal diffractometer D1B at the Institut Laue-Langevin, France $(\lambda=$ $2.529 \AA$ ).

\section{Results and discussion}

The X-ray diffraction on prepared sample proved hexagonal ZrNiAl-type structure unambiguously. The refinement of the structural parameters from diffraction patterns (at room temperature) was done using the Rietveld analysis employing the Fullprof program [10] leading to parameters: $a=7.71(1) \AA, c=4.08(1) \AA$, 
$x_{\mathrm{Ce}}=0.587(2), x_{\mathrm{In}}=0.247(2)$. The temperature evolution of lattice parameters was investigated employing D1B diffractometer. The parameters $a$ and $c$ as well as elementary unit volume decrease linearly with decrease of temperature down to $\approx 50 \mathrm{~K}$, see Fig. 1 . At lower temperatures, the lattice parameter $a$ remains almost constant. No evidence of structural phase transition is observed. Thermal expansion is well described by the change of unit cell volume with temperature: $V(T)=V_{0}\left[1+\beta\left(T-T_{0}\right)\right]$, where $V_{0}$ is the volume at $T_{0}$ and $\beta$ is the volume thermal expansion coefficient. Taking $T_{0}=40 \mathrm{~K}$ and thus $V_{0}=69.36 \AA^{3}$ we got $\beta=3.9 \times 10^{-5} \mathrm{~K}^{-1}$. The atomic positions in the unit cell, $x_{\mathrm{Ce}}$ and $x_{\mathrm{In}}$, remain constant with varying temperature.

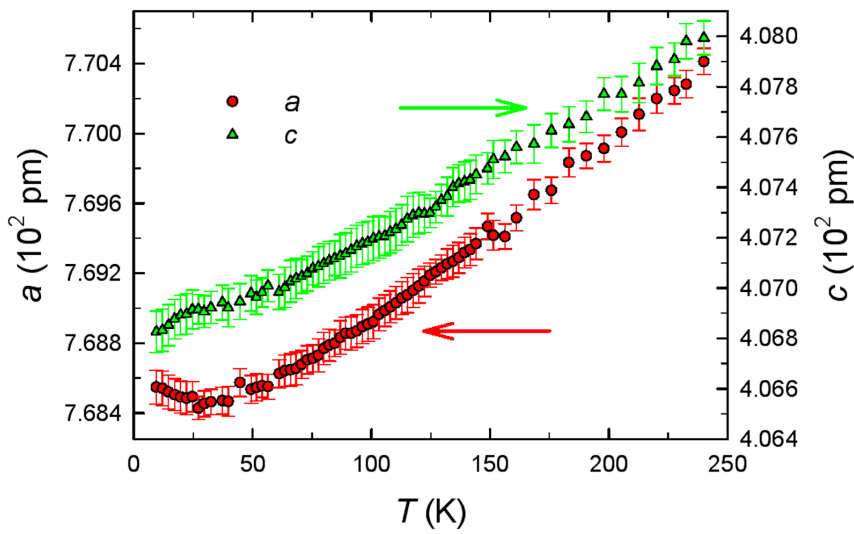

Fig. 1. Temperature evolution of lattice parameters measured using D1B neutron diffractometer at ILL.

In the paramagnetic region, the temperature dependences of magnetization (not shown) follow the CurieWeiss law. The obtained value of effective magnetic moment, $\mu_{\text {eff }}=2.59(2) \mu_{\mathrm{B}}$ for magnetic field oriented both parallel and perpendicular to the $c$-axis, is very close to the $\mathrm{Ce}^{3+}$ free ion value of $2.54 \mu_{\mathrm{B}}$. The negative paramagnetic Curie temperatures, $\theta_{p}=-34.1$ and $-56.8 \mathrm{~K}$, respectively, indicate antiferromagnetic ground state in CePdIn. These data are generally well in agreement with Ref. [1], although, the values of $\theta_{p}$ obtained by our measurements are somewhat lower than those in Ref. [1]. Nevertheless, the measure of the anisotropy of the paramagnetic Curie temperature $\Delta \theta_{p}=22.7 \mathrm{~K}$ remains practically the same $\left(\Delta \theta_{p}=22.0 \mathrm{~K}[1]\right)$. The magnetic field dependence of magnetization was measured as well. The magnetic field was applied along both principal directions leading to very similar results as obtained for isostructural CePtIn [6]. The value of magnetization measured at $1.8 \mathrm{~K}$ and in $7 \mathrm{~T}$ reaches value of $0.41 \mu_{\mathrm{B}} / \mathrm{f} . \mathrm{u}$. and seems to further increase with increase of field.

The temperature dependence of electrical resistivity develops fully in agreement with previously published data [1]. In this work, we focus on the low-temperature development of electrical resistivity in magnetic fields (Fig. 2). The broad anomaly on resistivity data around $3 \mathrm{~K}$ can be attributed to the Kondo effect. Another kink at around 1.7(2) $\mathrm{K}$ corresponds to the antiferromagnetic phase transition as revealed from specific heat measurement [3]. No anomaly around $0.9 \mathrm{~K}$ was observed in contrast to specific heat measurement [3]. The electrical resistivity value at low temperatures is significantly lowered by applied magnetic field (Fig. 2) as the field influences the magnetic moments of Ce atoms and presumably aligns them along the field direction.

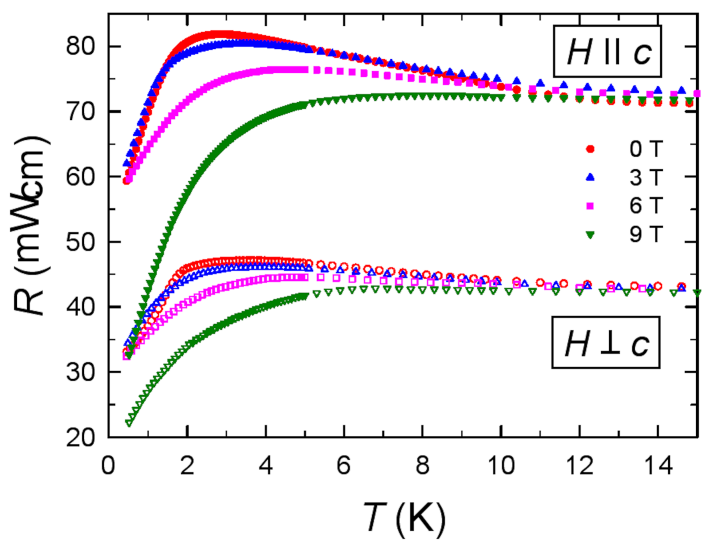

Fig. 2. Temperature development of electrical resistivity in low-temperature region and in several magnetic fields.

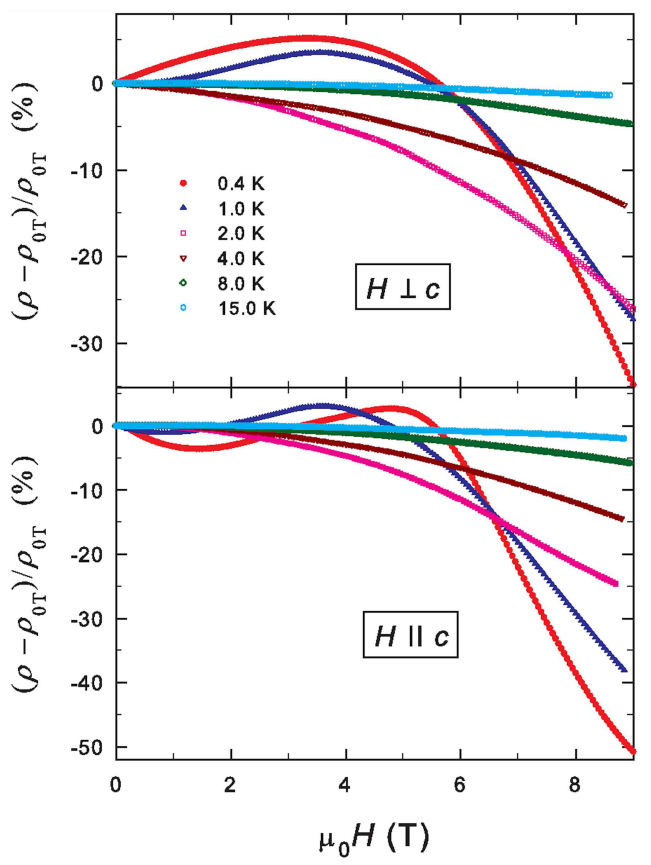

Fig. 3. Magnetic field dependence of electrical resistivity measured at temperatures below and above $T_{N}$.

The magnetoresistance (MR) data are shown in Fig. 3. The measurements with magnetic field along and perpendicular to the $c$-axis at $0.4 \mathrm{~K}$ reveal quite different behavior. The measurement for $H \perp c$ exhibits a small positive magnetoresistance in magnetic fields up to $4.5 \mathrm{~T}$ and a negative MR in higher fields (around $-35 \%$ in field 
of $9 \mathrm{~T}$ ). The negative MR, which turns to positive one in $\approx 3 \mathrm{~T}$ field, is present for arrangement with $\boldsymbol{H} \| \boldsymbol{c}$. In magnetic fields higher than $5 \mathrm{~T}$ the negative magnetoresistance is observed (down to $-50 \%$ in $9 \mathrm{~T}$ field). The above described behavior is observed for MR curves measured at temperatures below $T_{N}$. The suppression of the Kondo scattering with increasing field as well as the influence of field on the RKKY interaction could be marked as the origin of observed behavior. In the paramagnetic state, the regular development with negative MR is observed.

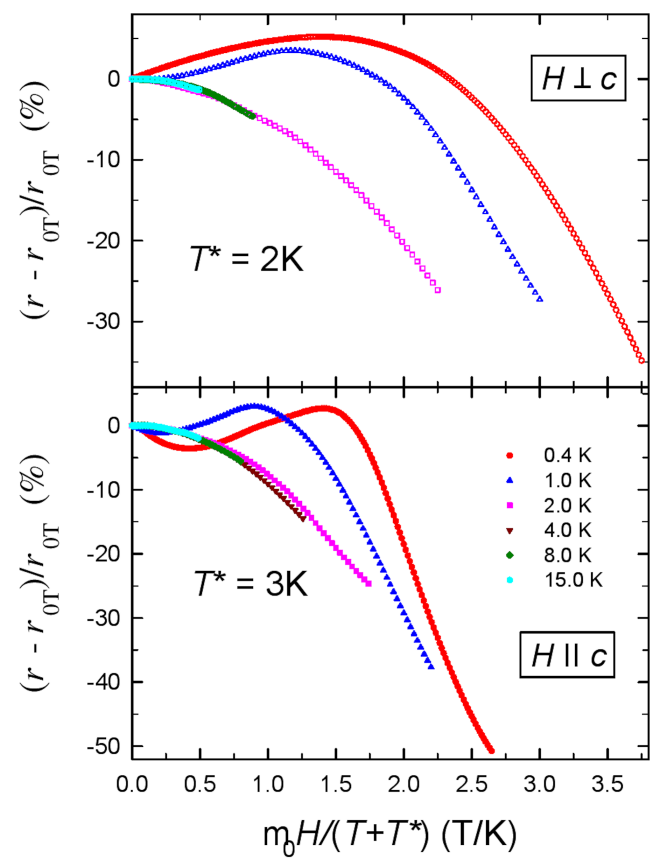

Fig. 4. Magnetoresistance measurement. The $x$-axis was rescaled [12-14]. $T^{*}$ is a measure of a strength of the ferromagnetic correlations in the system.

We followed the approach used in Refs. [11-13] taking into account the single-ion Kondo model with Betheansatz studies. Figure 4 was obtained by rescaling the $x$-axis of Fig. 3, using $T^{*}$ as a measure of the strength of ferromagnetic correlations in given compound $[12,13]$. $T^{*}$ is obtained in a way to get a significant overlap of MR curves measured in the paramagnetic state, i.e. at 2, 4, 8, and $15 \mathrm{~K}$ (Figs. 3 and 4 ). The value of $T^{*}$ is positive and relatively high for both measured directions, no or only weak ferromagnetic correlations between Ce moments in paramagnetic state are thus expected.

The powder neutron diffraction data measured employing E6 diffractometer at $0.4 \mathrm{~K}$ did not revealed any magnetic peak in diffraction patterns. Therefore the magnetic structure in CePdIn remains unknown. On the basis of neutron diffraction experiment, we can estimate the magnetic moment on Ce atoms to be significantly lower than $0.5 \mu_{\mathrm{B}}$. Such a moment is well in agreement with results of magnetization measurements. The single crystal neutron diffraction represents a possible way how to determine the magnetic structure of CePdIn and it is highly desirable.

\section{Conclusions}

CePdIn single crystal was studied by means of magnetization and electrical resistivity measurements. The magnetic ordering temperature was found to be $1.7 \mathrm{~K}$ in accordance with previous studies. The powder neutron diffraction studies did not reveal any magnetic peak at temperatures down to $0.4 \mathrm{~K}$, the magnetic moment on Ce atoms is estimated to be lower than $0.5 \mu_{\mathrm{B}}$. The single crystal neutron diffraction study is desirable to reveal the ground state magnetic structure.

\section{Acknowledgments}

The most of experiments were performed in MLTL $(/ / \mathrm{mltl} . \mathrm{eu} /)$, which is supported within the program of Czech Research Infrastructures (project no. LM2011025).

The work was supported within the program of Large Infrastructures for Research, Experimental Development and Innovation (Project No. LM2015050) and research project LTT17019 financed by the Ministry of Education, Youth and Sports, Czech Republic.

We would like to acknowledge Helmholz Zentrum Berlin. This research project has been supported by the 7th Framework Programme through the "Research infrastructures" action of the "Capacities" Programme, CP-CSA_INFRA-2011-1.1.17 Number 233883 NMI3 II.

\section{References}

[1] H. Fujii, T. Takabatake, Y. Andoh, J. Alloys Comp. 181, 111 (1992).

[2] H. Fujii, T. Inoue, Y. Andoh, T. Takabatake, K. Satoh, Y. Maeno, T. Fujita, J. Sakurai, Y. Yamaguchi, Phys. Rev. B 39, 6840 (1989).

[3] K. Satoh, Y. Maeno, T. Fujita, Y. Uwatoko, H. Fujii, J. Phys. (France) C8, 779 (1988).

[4] H. Fujii, M. Nagasawa, H. Kawanaka, T. Inoue, T. Takabatake, Physica B 165-166, 435 (1990).

[5] T. Fujita, K. Satoh, Y. Maeno, Y. Uwatoko, H. Fujii, J. Magn. Magn. Mater. 76-77, 133 (1988).

[6] M. Klicpera, P. Javorský, Mater. Res. Express 1, 016301 (2014).

[7] E. Brück, H. Nakotte, K. Bakker, F. de Boer, P. de Chatel, J. Li, J. Kuang, F. Yang, J. Alloys Comp. 200, 79 (1993).

[8] M. Klicpera, P. Javorský, E. Šantavá, J. Phys. Condens. Matter 25, 245501 (2013).

[9] T. Fujita, T. Suzuki, S. Nishigori, T. Takabatake, H. Fujii, J. Sakurai, J. Magn. Magn. Mater. 108, 35 (1992).

[10] J. Rodriguez-Carvajal, Physica B 192, 55 (1993).

[11] P. Schlottmann, Z. Phys. B Condens. Matter 51, 223 (1983).

[12] D.P. Rojas, J. Rodríguez Fernandez, J.I. Espeso, J.C. Gómez Sal, J. Alloys Comp. 502, 275 (2010).

[13] R. Pietri, B. Andraka, D. Kaczorowski, A. LeitheJasper, P. Rogl, Phys. Rev. B 61, 12169 (2000). 